Bio-grafía Escritos sobre la Biología y su Enseñanza.

Edición Extra-Ordinaria.

Memorias del 1 Congreso Nacional de Investigación en Enseñanza de la Biología.

VI Encuentro Nacional de Investigación en Enseñanza de la Biología y la

Educación Ambiental. ISSN 2027 1034. P. P. 103-115.

\title{
CIENCIA PARA NO CIENTÍFICOS: LO QUE EINSTEIN DESCONOCÍA SOBRE EL CAMBIO CLIMÁTICO
}

\section{SCIENCE FOR NO SCIENTISTS: WHAT EINSTEIN DID NOT KNOW ABOUT CLIMATE CHANGE}

\section{POR : Yair Alexander Porras Contreras ${ }^{1}$ Ivonne Inés Angarita Gálvez}

\section{Resumen}

Ante el agravamiento de la crisis ambiental global, la Ciencia y la Tecnología se han convertido en uno de los referentes para la solución de problemáticas tan diversas como la pobreza, la inseguridad alimentaria y el cambio climático, entre otras, por lo que su influencia en la transformación de la sociedad, constituye uno de los retos para la formación ciudadana. Consecuentemente, el estudio de las relaciones Ciencia, Tecnología, Sociedad y Ambiente (CTSA), que pretende examinar la situación del planeta, las desigualdades e inequidades que imperan en el mundo globalizado, con el objeto de empoderar a los ciudadanos en la toma de decisiones, plantea una paradoja en la cual se atribuye a la ciencia y la tecnología, también la responsabilidad de la aparición e incremento de dichas problemática ambientales. Ante esta disyuntiva, el proyecto CIENCIA PARA NO CIENTÍFICOS desarrollado por el Club de Astronomía y Ciencias "TATAXUE", conformado por un grupo de docentes y estudiantes del Colegio Distrital Orlando Fals Borda (Bogotá-Colombia), pretende desmitificar el papel de la Ciencia y la Tecnología como los únicos conocimientos válidos y confiables con los cuales las sociedades pueden hacer frente a las amenazas ambientales, lo cual plantea una crítica tanto a la representación social "del alumno como científico" (Marín y Cárdenas, 2011), como al movimiento de "alfabetización científica y tecnológica" (Gil y Vilches, 2005), por considerarlos excluyentes y propugnar una representación universalista del conocimiento. Este proyecto ha consolidado la participación de la comunidad educativa hacia el reconocimiento de acciones sustentables con el ambiente y la investigación en el marco de la construcción de múltiples ciudadanías. De igual manera, el proceso metodológico interdisciplinar que se construye al interior del propósito formativo, considera cuatro líneas de investigación 1) Astrobiología, 2) Biofilia 3) ReutilizARTE y 4) Ceres S'cool, en las cuales se desarrollan estrategias didácticas que permiten conocer las causas y consecuencias del cambio climático, permitiendo adelantar acciones en

\footnotetext{
${ }^{1}$ Docente de Ciencias Naturales y Educación Ambiental Colegio Orlando Fals Borda. Magister en Docencia de la Química. yairporras@yahoo.es. Docente catedrático UPN.

${ }^{2}$ Docente de Preescolar Colegio Orlando Fals Borda. Candidata a Magister en Educación. iangaritag@gmail.com
} 
Bio-grafía Escritos sobre la Biología y su Enseñanza.

Edición Extra-Ordinaria.

Memorias del 1 Congreso Nacional de Investigación en Enseñanza de la Biología.

VI Encuentro Nacional de Investigación en Enseñanza de la Biología y la

Educación Ambiental. ISSN 2027 1034. P. P. 103-115.

torno a la adaptación frente a esta problemática global. Los resultados de este trabajo se recogen en un libro electrónico elaborado por el IDEP con asesoría de la Pontificia Universidad Javeriana.

Palabras Clave: Cambio Climático, Ciencia, Tecnología, Sociedad, Ambiente

\section{Abstract}

Given the aggravation of the global environmental crisis, Science and Technology have become a concerning for the solution of diverse problems such as poverty, food insecurity and climate change, among others, so their influence on the transformation of society, is one of the challenges for citizenship education. Consequently, the study of relationships Science, Technology, Society and Environment (STSE), which aims to examine the situation in the world, inequalities and inequities that prevail in the globalized world, in order to empower citizens in decision-making raises a paradox which is attributed to science and technology, also the responsibility of the emergence and growth of these environmental problems.

In view of this dilemma, the project that is called "Science for no scientists" was developed by the Club of Astronomy and sciences "TATAXUE" made up by a group of Orlando Fals Borda School( Bogotá- Colombia) teachers and students and it aims at demystifying the role of Science and Technology as the only valid and reliable knowledge which societies can cope with environmental threats, which poses a critique of both social representation "of the student as a scientist" (Marin and Cárdenas, 2011), and the movement of "scientific and technological literacy" (Gil and Vilches, 2005), considering and advocating them exclusive and proposing an universalist representation of knowledge.

This project has consolidated the educational community participation towards the recognition of sustainable actions and research in the context of the construction of multiple citizenships. Likewise, the interdisciplinary methodological process that is built inside the formative purpose considers four research lines 1) Astrobiology, 2) Biophilia 3) Reuse ART and 4) Ceres S'cool, in which didactic strategies are developed that provide insight the causes and consequences of climate change, allowing further actions regarding the adaptation to this global problem. The results of this work are contained in an electronic book made by IDEP with advice from the Pontificia Universidad Javeriana.

Key words: Climate change, Science, Technology, Society, Environment 
Bio-grafía Escritos sobre la Biología y su Enseñanza.

Edición Extra-Ordinaria.

Memorias del 1 Congreso Nacional de Investigación en Enseñanza de la Biología.

VI Encuentro Nacional de Investigación en Enseñanza de la Biología y la

Educación Ambiental. ISSN 2027 1034. P. P. 103-115.

\section{INTRODUCCIÓN}

A diario leemos y escuchamos noticias sobre el cambio climático y somos testigos de la variabilidad en las condiciones meteorológicas, las cuales de manera errática se alejan de algunos patrones estadísticos analizados durante años. De hecho, la presencia de fenómenos naturales como "El Niño" y "La Niña", que provocaron en el año 2010 un aumento de la temperatura terrestre en $0,55^{\circ} \mathrm{C}$, y en el año 2011 , la peor ola invernal en la historia de Colombia, son asociadas frecuentemente con el cambio climático y con conductas humanas insostenibles, que continúan acabando los recursos naturales e influyen en el equilibrio ecológico del planeta. Desde el punto de vista científico, este aumento de la temperatura global, deja al año 2010 como uno de los más caóticos en la historia reciente del planeta, de acuerdo con la proyección climatológica adelantada por el Instituto Goddard para Estudios Espaciales de la NASA ${ }^{3}$. En el caso particular de Usme, el seguimiento a los discursos locales por medio de entrevistas relacionadas con el impacto de los eventos meteorológicos en la vida de las personas, evidenció una manera de asimilar la variabilidad climatológica con conductas insostenibles que han desprovisto de recursos naturales una amplio sector de la ciudad, lo que ha ocasionado deslizamientos y derrumbes en los cerros orientales, específicamente en los barrios aledaños al colegio, donde habitan los estudiantes o tienen familiares. Por estas razones, el tema de los desplazamientos humanos por causa del conflicto armado o por eventos climatológicos, debe ser analizado con detenimiento por las autoridades pertinentes.

Hacer frente a la pobreza requiere mejorar el poder de participación y decisión de las personas en aspectos relevantes para sus vidas, como lo son las condiciones de bienestar. De hecho, existe un amplio sector de la población que duda sobre la existencia de un cambio climático o, que si bien acepta que existe, considera que el planeta por sí sólo, tiene la capacidad de reciclar aquello que contamina. Por tal razón, las preguntas iniciales con las cuales adelantamos nuestra propuesta de innovación fueron: ¿Existe el cambio climático? ¿Si es así cómo podemos evidenciarlo? ¿Qué impactos genera? ¿Cómo podemos detenerlo? De esta manera, decidimos consultar documentos relacionados con las cumbres internacionales sobre cambio climático (IPCC, 2007; UNFCCC. 2007), investigaciones relacionadas con sus causas e impactos (Rosenzweig et al, 2008; Karl et al, 2009; Cardona, A. 2009), al igual que la opinión de educadores ambientales a nivel mundial (UNESCO, 2004; Rule y Meyer, 2009; Mackeown y Hopkins, 2010; Dent y Dalton, 2010; Punter et al, 2011). En todos estos casos vimos la necesidad de involucrar diferentes discursos en el análisis de la situación ambiental, articulando el tema del cambio climático a la instalación de diversas

${ }^{3}$ Disponible en http://data.giss.nasa.gov/gistemp/2010july/ 
Memorias del 1 Congreso Nacional de Investigación en Enseñanza de la Biología.

VI Encuentro Nacional de Investigación en Enseñanza de la Biología y la

Educación Ambiental. ISSN 2027 1034. P. P. 103-115.

crisis que atraviesan la educación, la calidad de vida y el concepto de bienestar. Así concluimos que estas amenazas globales no pueden ser analizadas sin tener en cuenta variables como el crecimiento demográfico, la disminución de las tierras de cultivo, la inestabilidad climática, las fuentes energéticas y, por sobre todo, las precarias condiciones sociales que impiden la solidaridad, la cooperación, la conformación de redes de trabajo y la puesta en marcha de proyectos que promuevan la apropiación social de la Ciencia y la Tecnología y la valoración de los saberes locales.

De acuerdo con estos argumentos, creemos firmemente que la escuela no puede ser ajena a estas problemáticas, por el contrario debe enfocarse en incrementar las opciones de los miembros de la comunidad educativa para construir un proyecto de vida, que combata la pobreza, en cuanto a la negación de oportunidades y contribuya a la consolidación de una ciudadanía ambientalmente responsable. En este sentido, el proyecto CIENCIA PARA NO CIENTíFICOS: LO QUE EINSTEIN DESCONOCÍA SOBRE EL CAMBIO CLIMÁTICO, es un trabajo de innovación pedagógica porque se constituye una práctica social dialógicamente construida que pretende institucionalizar una experiencia exitosa centrada en el reconocimiento de acciones sostenibles, fruto de la investigación y la resolución de problemáticas ambientales, las cuales fomentan la preparación para la acción y la toma de decisiones colectivas, en un mundo cada vez más cambiante y más complejo.

\section{METODOLOGÍA}

Dado el carácter de la investigación, el diseño planteado desde el paradigma cualitativo, busca la transformación de las prácticas pedagógicas y la resolución de problemáticas educativas. La propuesta se considera una construcción colectiva basada en la auto-reflexión, la cual permite hacer visibles los procedimientos, las estrategias, los contenidos con sus aciertos y desaciertos, para a partir de su visibilización y evaluación, plantear alternativas pedagógicas y didácticas, desde la Investigación-Acción-Participación. De acuerdo con lo anterior, compartimos con Ull et al (2009) el manejo de tres competencias para la sostenibilidad:

- Competencias cognitivas relacionadas con el saber y vinculadas con la comprensión crítica de la problemática ambiental global y local;

- Competencias metodológicas, relacionadas con el saber-hacer, la adquisición de habilidades, estrategias, técnicas y procedimientos para la toma 
Memorias del 1 Congreso Nacional de Investigación en Enseñanza de la Biología. VI Encuentro Nacional de Investigación en Enseñanza de la Biología y la Educación Ambiental. ISSN 2027 1034. P. P. 103-115.

de decisiones y la realización de acciones relacionadas con el medio ambiente y el desarrollo sostenible;

- Competencias actitudinales, relacionadas con el saber ser y valorar, donde el desarrollo de actitudes y valores de sostenibilidad resulta imprescindible.

POBLACIÒN: Los 40 estudiantes del club, los 5 padres de familia y los 4 profesores, se reúnen en jornada contraria, en un seminario permanente, los días miércoles de 9:30 a 12:00, en las instalaciones del colegio.

\section{CICLO DE INVESTIGACIÓN “TATAXUE”}

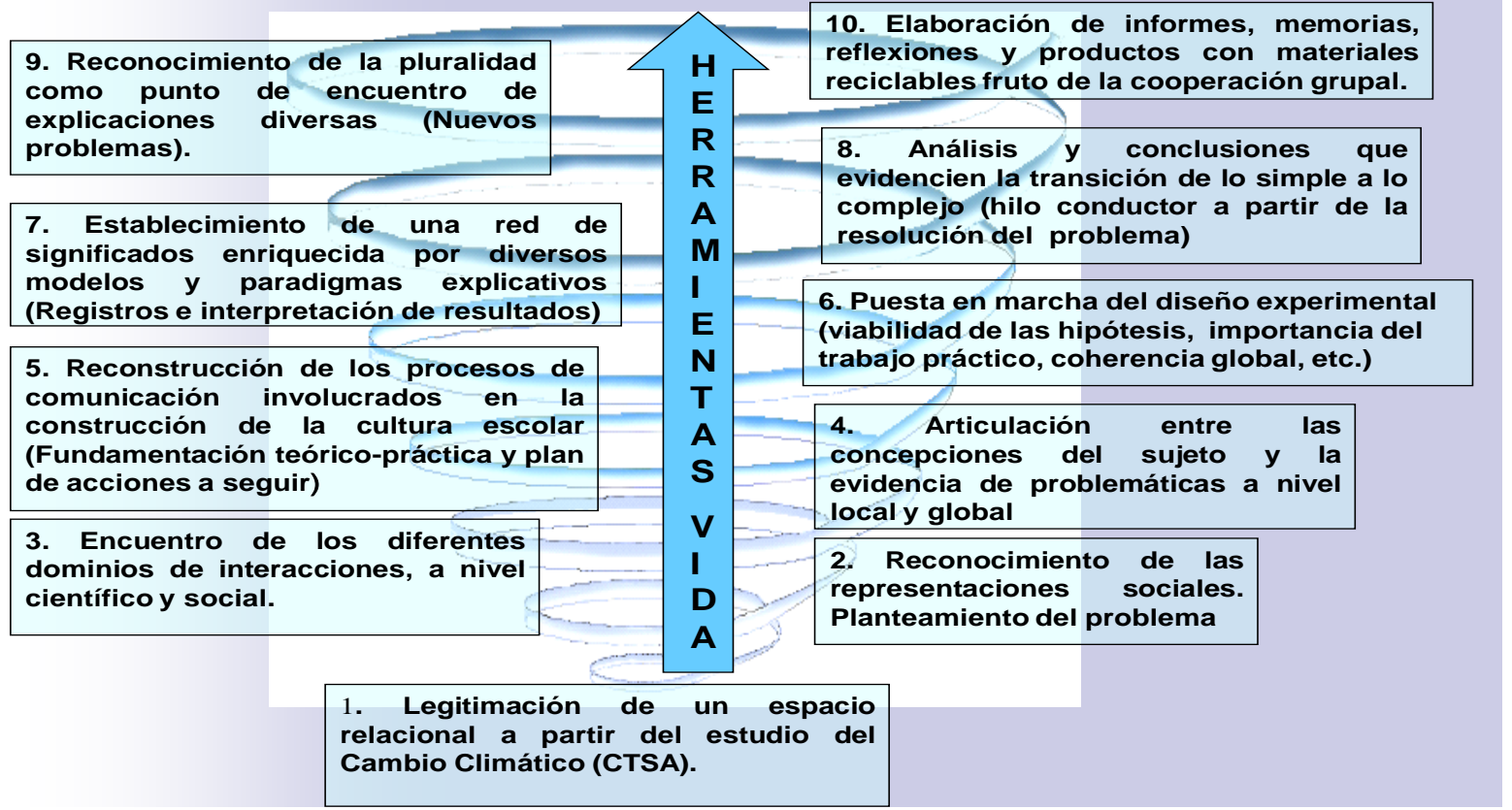

Esquema 1. Ciclo de Investigación del Club de Astronomía y Ciencias "Tataxue". 
Bio-grafía Escritos sobre la Biología y su Enseñanza.

Edición Extra-Ordinaria.

Memorias del 1 Congreso Nacional de Investigación en Enseñanza de la Biología.

VI Encuentro Nacional de Investigación en Enseñanza de la Biología y la

Educación Ambiental. ISSN 2027 1034. P. p. 103-115.

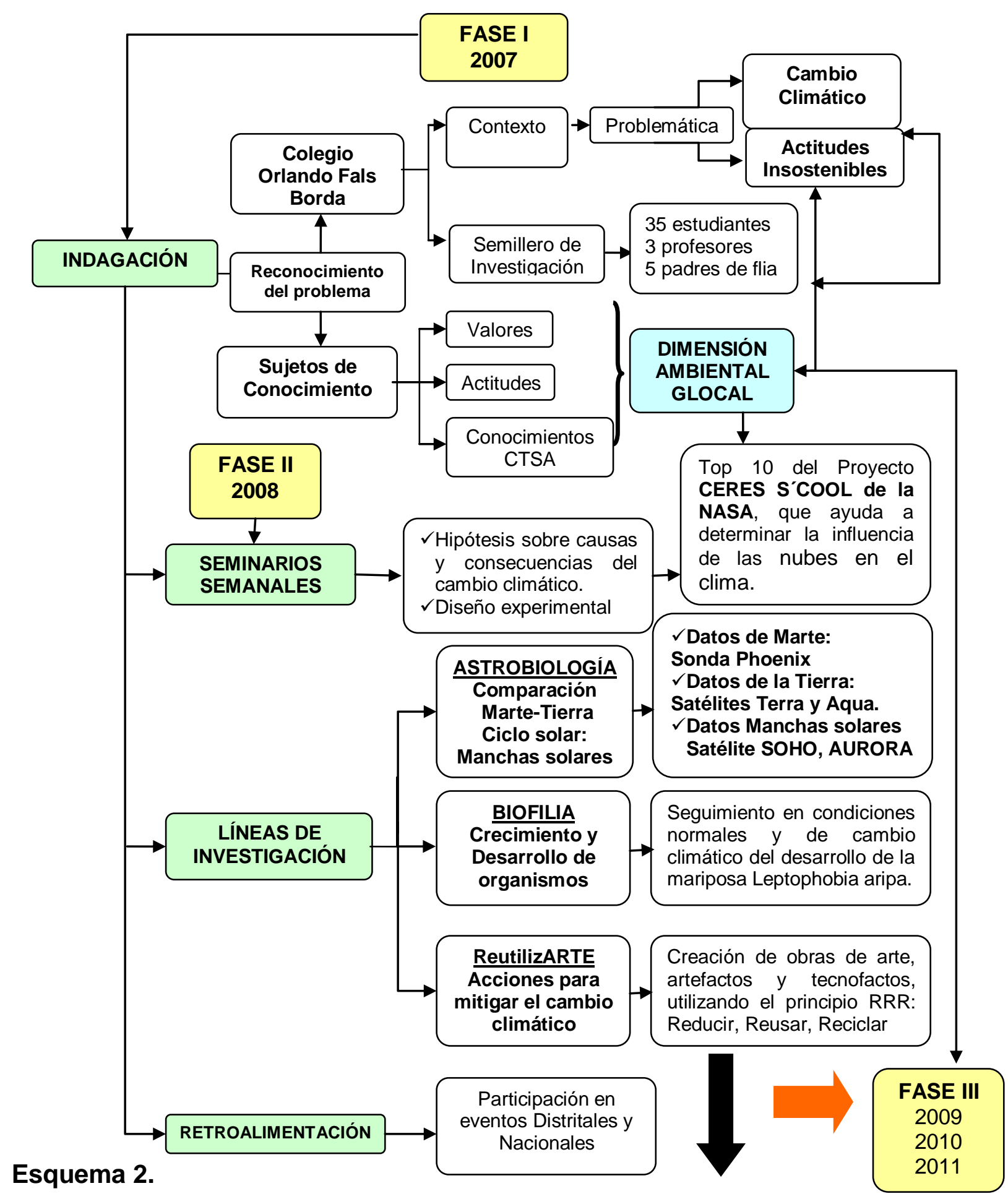


Memorias del 1 Congreso Nacional de Investigación en Enseñanza de la Biología. VI Encuentro Nacional de Investigación en Enseñanza de la Biología y la Educación Ambiental. ISSN 2027 1034. P. P. 103-115.

\section{RESULTADOS Y DISCUSIÓN}

1. Diagnóstico: En esta etapa se reconocieron las características del grupo de estudiantes, su realidad familiar, escolar y local, así como sus intereses y expectativas con relación al tema de estudio. Igualmente, se indagaron algunas representaciones sociales de ambiente, a través de un cuestionario, contando con la subsecuente observación directa y cercana del grupo, orientando la propuesta didáctica a la legitimación de los intereses del grupo. Las representaciones sociales se constituyeron en el referente para comprender cómo las personas adelantan la construcción de un sistema de ideas particulares para interpretar, entender la realidad y vincularse al grupo social. En el caso particular del cambio climático, es pertinente decir que las representaciones sociales evidenciadas involucran aspectos significativos de la realidad, con los cuales se construyó un lenguaje común y algunos referentes de actuación para dar resolución a la problemática en cuestión. En los años 2010 y 2011 el trabajo de indagación se profundiza con el registro audiovisual a través del video "Tataxue: el legado de los hijos de las estrellas" auspiciado por la Corporación Maloka. De igual manera, el intercambio de ideas a través de las redes sociales ha consolidado una manera particular de establecer un contacto permanente con estudiantes y profesores, garantizando la comunicación en tiempo real y el aprovechamiento de los recursos tecnológicos bien sea en facebook, twitter, el blog del club o la página web del colegio.

2. Diseño: A partir de las representaciones encontradas en la fase de diagnóstico, surgieron preguntas que se enfocaron en: reconocer la variabilidad climatológica en términos de años, discutir la existencia del cambio climático en la actualidad, formular hipótesis sobre la manera de estudiar el clima y reconocer su impacto sobre los seres vivos. De esta manera, la realización de seminarios donde se abordaron temáticas relacionadas con el ambiente desde una perspectiva sistémica, la educación ambiental, el cambio climático, los semilleros de investigación y la importancia de estudiar el contexto local, permitieron delimitar el problema de investigación y formular alternativas pedagógicas y didácticas para adelantar un proceso de investigación educativa. En esta fase se constituyeron las líneas de investigación que tienen por objeto solucionar una problemática específica dentro del programa de formación ambiental enfocado al estudio del cambio climático. Cada línea tiene un estudiante líder que dinamiza las discusiones y 
Memorias del 1 Congreso Nacional de Investigación en Enseñanza de la Biología. VI Encuentro Nacional de Investigación en Enseñanza de la Biología y la Educación Ambiental. ISSN 2027 1034. P. P. 103-115.

organiza las actividades por sesión, con la asesoría de los docentes coordinadores.

3. Implementación: En esta fase se formularon tres investigaciones, con el objeto de estudiar las causas y consecuencias del cambio climático, las cuales continúan implementándose y profundizándose en el Club "Tataxue", de acuerdo a los nuevos hallazgos y enfoques que se han establecido por consenso. Así, la comparación entre las condiciones climáticas de Marte y la Tierra, al igual que el estudio de las manchas solares como fenómenos que influyen tanto en el ciclo solar, el clima de la Tierra, como en la probabilidad de vida (Gray et al. 2010), son estudiados por la línea de investigación ASTROBIOLOGÍA; el seguimiento al ciclo de vida de las mariposas, por constituirse en bioindicadores de calidad ambiental y organismos sensibles al cambio climático (Andrade, 1998), es estudiado por la línea de investigación BIOFILIA, en la cual se ha realizado un seguimiento al ciclo de vida de la mariposa "Leptophobia aripa" y actualmente se enfoca en el diseño y construcción del mariposario automatizado para la cría de especies como Dannaus plexipus (monarca). Este subproyecto es orientado por el programa Ondas de Colciencias, en la línea de Robótica y automatización y en él se viene estudiando, a su vez la manera de garantizar la siembra de la planta hospedera Asclepia, por medio de cultivos in vitro. De igual manera la relación arte-ciencia-sustentabilidad (Malina, 2009) se evidencia con el desarrollo de acciones creativas, en las que se reutilicen, reduzcan y reciclen algunos materiales (reutilizARTE) (Anexo 1).

4. Evaluación: En esta etapa se retroalimenta el proceso, identificando los progresos, los avances en los objetivos propuestos, los aciertos y desaciertos del proceso investigativo. Aquí se tienen en cuenta los resultados de cada línea de investigación, al igual que los informes de los estudiantes, los cuales son analizados desde una mirada sistémica de ambiente. Parte del proceso de retroalimentación se adelantó en el año 2011 con la sistematización de la experiencia, a partir de la orientación del IDEP y la Universidad Javeriana. EI resultado del trabajo de sistematización es el libro "Ciudadanos ante el Cambio Climático: Un Proyecto de Vida Compartido" publicado por el IDEP en el año 2011. De igual manera, una manera de evaluar la propuesta es colocándola al escrutinio público, mediante la participación en programas como "Maestros que Aprenden de Maestros", Simposios, Ferias Pedagógicas Institucionales, Locales y Distritales, Expociencia Juvenil, Campamentos Espaciales, entre otros. Adicionalmente, en el año 2010 se desarrolla el trabajo de grado denominado "La Enseñanza del Cambio Climático por Medio de una Estructura Problematizada: Una Estrategia Didáctica para Desarrollar 
Bio-grafía Escritos sobre la Biología y su Enseñanza.

Edición Extra-Ordinaria.

Memorias del 1 Congreso Nacional de Investigación en Enseñanza de la Biología.

VI Encuentro Nacional de Investigación en Enseñanza de la Biología y la

Educación Ambiental. ISSN 2027 1034. P. P. 103-115.

Competencias Científicas en Estudiantes del Colegio Orlando Fals Borda", por parte de la estudiante Nataly Soto en el marco de la línea de Investigación "Educación Ambiental en el Contexto Educativo Colombiano" del Departamento de Biología de la Universidad Pedagógica Nacional.

\section{CONCLUSIONES}

Es claro que la formación académica y personal que se desarrolla a nivel escolar no garantiza el estudio de las relaciones Ciencia, Tecnología, Sociedad y Ambiente, desde una perspectiva compleja, ya que los procesos formativos que se adelantan en las instituciones educativas olvidan, en muchas ocasiones, las interacciones que a nivel social los sujetos establecen a partir de su subjetividad; por lo que aquellos esquemas de interpretación del mundo (personal, científico, filosófico, ético, estético, etc.) determinados por las representaciones que se construyen, se pasan por alto en la formación docente. Teniendo en cuenta estos planteamientos, complejizar la dinámica educativa tomando como eje de reflexión el estudio del Cambio Climático, parte de un desconocimiento general de la crisis ambiental global por parte de la comunidad educativa, el cual se relaciona con un precario tratamiento de la naturaleza de la ciencia y la tecnología, siendo las representaciones más recurrentes aquellas que sugieren una ciencia libre de valores morales, una tecnología como ciencia aplicada y un ambiente reducido a aspectos ecológicos, las cuales en conjunto pueden influir en la propia práctica profesional futura.

El entendimiento de los riesgos, la vulnerabilidad, y las oportunidades de actuación para enfrentar las problemáticas ambientales, desde una perspectiva sistémica, son quizás los retos más importantes de los acuerdos internacionales para enfrentar el cambio climático en el siglo XXI. De hecho, la creciente atención a los contenidos en ciencia y tecnología en los procesos formativos, su posible impacto en los aspectos socio-ambientales y la importancia que se le brinda a los movimientos de alfabetización científica y tecnológica, ciencia para todos, apropiación social de la ciencia 0 , como consideramos en este escrito, "democratización de la ciencia y la tecnología", merecen ser estudiados con detenimiento, especialmente como derroteros por los cuales se construye un ideario de ciudadanía.

Estas demandas educativas colocan nuevos retos a la Educación en Ciencia y Tecnología, y un desafío superior a la Educación Ambiental, al convertirse en "una dimensión de la educación integral y global de las personas y colectividades sociales, que en sus diversas manifestaciones y prácticas, promueve el 
Bio-grafía Escritos sobre la Biología y su Enseñanza.

Edición Extra-Ordinaria.

Memorias del 1 Congreso Nacional de Investigación en Enseñanza de la Biología.

VI Encuentro Nacional de Investigación en Enseñanza de la Biología y la

Educación Ambiental. ISSN 2027 1034. P. P. 103-115.

conocimiento, interpretación y concienciación respecto de las diferentes problemáticas ambientales, de su impacto local y planetario, activando competencias y valores de los que se deriven actitudes y comportamientos congruentes con la ética ecológica que se precisa para participar en la construcción de un desarrollo humano sostenible" (Caride, 2001). Más aún estos procesos de formación apuntan a una educación Emancipadora, con lo que se sitúa la educación ambiental como "una vía de replanteamiento de nuestras relaciones con la biosfera, a la vez que un instrumento de transformación social y empoderamiento de los más débiles, todo ello con la meta final de conseguir sociedades más armónicas y equitativas (Novo, 2009)".

\section{NOTAS FINALES}

Utilizando el instrumento de Solbes y Vilches (2004) sobre las relaciones CTSA en la formación ciudadana, los estudiantes que hacen parte del club "Tataxue", han mostrado cambios significativos en:

1) La forma de plantear los problemas a los que se enfrenta hoy la humanidad, sus causas y las posibles medidas a adoptar.

2) La Comprensión del papel de la ciencia y la tecnología en la solución de los problemas.

3) La manera de reconocer la influencia de la sociedad y de intereses particulares en los objetivos de la ciencia y la tecnología.

4) la forma de valorar éticamente, los desarrollos que contribuyen a la satisfacción de necesidades humanas.

\section{- Nivel de impacto en la comunidad educativa:}

Como parte de la formación científica, tecnológica y ambiental del semillero de investigación del club de ciencias-astronomía-arte "TATAXUE", se consideran dentro de los impactos del proyecto de investigación los siguientes:

$\checkmark \quad$ Consolidación de un proyecto que tiene como epicentro la formación de una ciudadanía ambientalmente responsable y recoge de manera interdisciplinaria una propuesta de incorporación de la educación ambiental a la práctica educativa. Es necesario aclarar que el proyecto es sostenible porque se van profundizando los temas, por ejemplo la proyección comparativa entre Marte y la Tierra no se reduce únicamente a Usme, sino también al desierto de la Tatacoa, visitado por todo el club en Julio de 2009-2011, y a la Isla Gorgona en noviembre de 2009.

$\checkmark \quad$ Sistematización de la experiencia con la colaboración del IDEP y la Universidad Javeriana, a través de un libro electrónico próximo a publicarse.

$\checkmark \quad$ Campañas de difusión del proyecto a través de exposiciones itinerantes, feria de la ciencia y proyecciones, con el ánimo de sensibilizar a la comunidad sobre medidas a implementar para disminuir el impacto de conductas ambientalmente irresponsables. 
Memorias del 1 Congreso Nacional de Investigación en Enseñanza de la Biología.

VI Encuentro Nacional de Investigación en Enseñanza de la Biología y la Educación Ambiental. ISSN 2027 1034. P. P. 103-115.

$\checkmark$ Entrevista radial al profesor Yair Porras en el "Magazín Pedagógico Escuela País" del Instituto para la Investigación Educativa y el Desarrollo Pedagógico - IDEP, donde se describe el proyecto "Ciudadanos ante el Cambio Climático", la metodología desarrollada y las transformaciones generadas en la comunidad educativa.

$\checkmark \quad$ Participación en el proyecto internacional CERES S'COOL. El proyecto CERES S'COOL de la NASA tiene como finalidad determinar la influencia de las nubes sobre el clima del planeta. Más de 2000 colegios de todo el mundo registran datos diariamente de los tipos de nubes, temperatura, presión atmosférica y humedad con el fin de realizar un mapeo del cambio climático y asegurar la efectividad de los satélites climáticos Terra y Aqua. El club de astronomía "Tataxue" ha liderado este proyecto en los tres últimos años, quedando entre los 10 mejores observadores del mundo. Para verificar resultados consultar la página http://scienceedu.larc.nasa.gov/SCOOL/top10.html

\section{BIBLIOGRAFIA}

- Acevedo, J. A. (1996). La tecnología en las relaciones CTS. Una aproximación al tema. Enseñanza de las Ciencias, 14 (1), pp. 35-44.

- Acosta, A. y Martínez, E. (Comp.) 2008. Buen vivir. Una vía para el desarrollo. Quito: Abya-Yala.

- ANDRADE-C. M.G. 1998. Utilización de las Mariposas como Bioindicadoras del Tipo de Hábitat y su Biodiversidad en Colombia. Rev. Acad. Colomb. Cienc. 22(84): 407-421

- Caamaño, A. (ed.) (1995). La educación ciencia-tecnología-sociedad. (Monográfico). Alambique, 3.

- Cardona, A. 2009. Mapeo Institucional. Actores relacionados con el abordaje del cambio climático en Colombia. Proyecto Integración de riesgos y oportunidades del cambio climático en los procesos nacionales de desarrollo y en la programación por países de las Naciones Unidas. PNUD: Bogotá.

- Caride, J. A. y Meira, P. A. 2001. Educación ambiental y desarrollo humano. Barcelona: Ariel.

- Dent, P and Dalton, G. 2010. Climate Change and Profesional Surveying Programmes of Study. International Journal of Sustainability in Higher Education. Vol. 11 No. 3, 2010 pp. 274-291.

- Fals Borda, O. Reflexiones sobre la investigación participativa. CENAPRO. México, 1981.

- García-Canclini, N. 1990. Culturas Híbridas. Estrategias para entrar y salir de la modernidad. Ed. Grijalbo, México. 
Memorias del 1 Congreso Nacional de Investigación en Enseñanza de la Biología. VI Encuentro Nacional de Investigación en Enseñanza de la Biología y la Educación Ambiental. ISSN 2027 1034. P. P. 103-115.

- Gil-Pérez, D. y Vilches, A. (2005). Inmersión en la cultura científica para la toma de decisiones. ¿Necesidad o mito? Eureka (http://www.apaceureka.org/revista/), 2(3), 302-329.

- Gray, L. J., et al. 2010. Solar influences on climate. Reviews of Geophysics, 48, RG4001, pg 1-53

- IPCC, 2007: Cambio climático 2007: Informe de síntesis. Contribución de los Grupos de trabajo I, II y III al Cuarto Informe de evaluación del Grupo Intergubernamental de Expertos sobre el Cambio Climático [Equipo de redacción principal: Pachauri, R.K. y Reisinger, A. (directores de la publicación)].

- Karl, T., Melillo, J. and Peterson, T (eds.). 2009. Global Climate Change Impacts in the United States,. Cambridge University Press.

- Sanderson, K. 2007. Mars hots up - Dust storms and dark rocks are making the red planet hotter. Nature.

- Manassero, M. A. y Vázquez, A. (1998). Opinions sobre ciencia, tecnología $i$ societat. Palma de Mallorca: Conselleria d'Educació, Cultura i Esports.

- Manassero, M. A. y Vázquez, A. (2001). Actitudes y creencias de los estudiantes relacionadas con CTS, en Membiela, P. (coord.). La enseñanza de las ciencias desde la perspectiva Ciencia-Tecnología-Sociedad. Formación científica para la ciudadanía, pp. 169-162. Madrid: Narcea.

- Malina, F. Conectar arte y Ciencia es una Cuestión Urgente. (Recuperado, Agosto 2009). http://www.conaculta.gob.mx/?p=19377

- Marín, N. y Cárdenas, F. 2011. Valoración de los Modelos más Usados en la Enseñanza de las Ciencias Basados en la Analogía «el Alumno como Científico». Enseñanza de las Ciencias. Volumen 29. № 1.

- Mackeown, R. y HopKins, Ch. 2010. Rethinking Climate Change Education. Everyone wants it, but what is it?. Green Teacher, Vol 89 Pag 17-21

- Meira, P. (dir.). 2009. La Sociedad Ante el Cambio Climático: Conocimientos, Valoraciones y Comportamientos en la Población Española. Fundación Mapfre.

- Membiela, P. (coord.) (2001). La enseñanza de las ciencias desde la perspectiva Ciencia-Tecnología-Sociedad. Formación científica para la ciudadanía. Madrid: Narcea.

- Molina, A; Martínez, C; Mosquera C; Mojica L. 2009. Diversidad Cultural e implicaciones en la enseñanza de las ciencias: reflexiones y avances. Revista Colombiana de Educación. Universidad Pedagógica Nacional. No. 56. Pg. 107-123.

- Novo, M. 2009. La educación ambiental, una genuina educación para el desarrollo sostenible. Revista de Educación, número extraordinario 2009, pp. 195-217. 
Memorias del 1 Congreso Nacional de Investigación en Enseñanza de la Biología. VI Encuentro Nacional de Investigación en Enseñanza de la Biología y la Educación Ambiental. ISSN 2027 1034. P. P. 103-115.

- Perez, R. y Porras, Y. 2005. La Complejidad en el Marco de una Propuesta Pluriparadigmática. Revista Tecné, Episteme y Didaxis. Vol. 17. p.104 116 ,

- Punter, P., Ochando-Pardo, M. and Garcia, J. 2011. Spanish Secondary School Students' Notions on the Causes and Consequences of Climate Change. International Journal of Science Education, 33: 3, 447 - 464

- Rosenzweig, C., D. Karoly, M. Vicarelli, P. Neofotis, Q.G. Wu, G. Casassa, A. Menzel, T.L. Root, N. Estrella, B. Seguin, P. Tryjanowski, C.Z. Liu, S. Rawlins, and A. Imeson. 2008. Attributing Physical and Biological Impacts to Anthropogenic Climate Change. Nature, 453(7193): 353-357.

- Rule, A. and Meyer, M. 2009. Teaching Urban High School Students Global Climate Change Information and Graph interpretation skills using evidence from scientific literature. Journal of Geoscience Education; Nov 2009; 57, 5; pg. 335

- Santos, B. 2009. Una Epistemología del Sur. CLACSO Coediciones, Siglo Veintiuno Editores, Buenos Aires.

- Solbes, J. y Vilches, A. (1997). STS interactions and the teaching of physics and chemistry. Science Education, 81 (4), pp. 377-386.

- Solbes, J. y Vilches, A. (2001). Percepciones del alumnado de ESO y bachillerato acerca de las interacciones CTS. Enseñanza de las Ciencias, núm. extra, VI Congreso,pp. 27- 28.

- Solbes, J. y Vilches, A. (2004). Papel de las Relaciones Ciencia, Tecnología, Sociedad y Ambiente en la Formación Ciudadana. Enseñanza de las Ciencias, 2004, 22(3).

- UNFCCC. 2007. Report on the workshop on climate related risks and extreme events. Note by the secretariat. FCCC/SBSTA/2007/7. UNFCCC. Bonn, Germany. http://unfccc.int/resource/docs/2007/sbsta/eng/07.pdf>

- UNESCO. 2004. United Nations Decade of Education for Sustainable Development: Draft International Implementation Scheme, París.

- Ull, M.; Aznar, P.; Martinez, M.; Palacios, B. y Piñero, A. 2009. Competencias para la sostenibilidad y curricula universitarios. Enseñanza de las Ciencias, Número Extra VIII Congreso Internacional sobre Investigación en Didáctica de las Ciencias, Barcelona, pp. 2957-2960

- Vilches, A y Gil-Pérez, D. 2009. Una Situación de Emergencia Planetaria a la que Debemos y Podemos Hacer Frente. Revista de Educación. Número Extraordinario 2009, 101-122.

- WorldWatch Institute. 2009. El mundo ante el calentamiento global. La Situación del Mundo. Barcelona. CIP-Ecosocial (FUHEM) e Icaria. 